\title{
Variation in the Structure and Activity of Commercial Palladium on Carbon Catalysts
}

\author{
Conor J. Crawford, ${ }^{[a] *}$ Yan Qiao, ${ }^{[b, c]}$ Yequn Liu, ${ }^{[b, c]}$ Dongmei Huang, ${ }^{[b, c]}$ Wenjun Yan,,${ }^{[b, c]}$ \\ Stefan Oscarson, ${ }^{[\mathrm{a}]^{*}}$ and Shuai Chen, ${ }^{[\mathrm{c}]^{*}}$
}

[a] Centre for Synthesis and Chemical Biology, University College Dublin, Belfield, Dublin, Ireland. E-mail: conor.crawford@ucdconnect.ie stefan.oscarson@ucd.ie

[b] Center of Materials Science and Optoelectronics Engineering, University of Chinese Academy of Sciences, Beijing 100049, People's Republic of China.

[c] State Key Laboratory of Coal Conversion, Institute of Coal Chemistry, Chinese Academy of Sciences, Taiyuan 030001, People's Republic of China. Email: chenshuai@sxicc.ac.cn qiaoy@sxicc.ac.cn

\begin{abstract}
Palladium catalysed hydrogenolysis is often the final step in challenging natural product total synthesis and a key step in industrial processes towards fine chemicals. Here we demonstrate that there is wide variability in quality of commercial sources of palladium on carbon $(\mathrm{Pd} / \mathrm{C})$, which results in differences in selectivity, reaction times, and yields. This is established through a combination of XRD, XPS, BET and TEM analysis, this will serve as a template for others to quickly identify a high-quality source of $\mathrm{Pd} / \mathrm{C}$ catalyst - meaning less time spent on time consuming reaction optimisation studies. Key indicators of a high-quality catalyst include: small particle sizes, large surface area, and the presence of both $\mathrm{Pd}^{0}$ and $\mathrm{Pd}^{2+}$ active species (not present in all commercial sources). Further this work will enable the design and synthesis of new high-quality $\mathrm{Pd} / \mathrm{C}$ catalysts.
\end{abstract}

\section{Introduction}

Palladium catalysed hydrogenolysis is often the penultimate step in challenging total synthesis to remove ether style protecting groups (e.g. benzyl or naphthylmethyl ethers) with the aim to yield the desired target compound. While deceivingly simplistic, this final step can be a major bottleneck. This challenge can be encountered when deprotecting synthetic glycans (particularly oligosaccharides), as the requirement to simultaneously remove large numbers of benzyl groups ( $>30$ groups) in high yields, high selectivity, and short times requires a high-quality source of $\mathrm{Pd} / \mathrm{C}$ catalyst. Further complicating this is the transformation of a highly lipophilic molecule to a hydrophilic one, which poses a range of solubility issues. This challenge can be demonstrated through the recent works of the $\mathrm{Yu}$ group, where their impressive total synthesis of a 128mer ${ }^{[1]}$ ended with a $15 \%$ yield in the hydrogenolysis reaction, or those of the Seeberger group in their report in the automated glycan assembly of Lewis type antigens, ${ }^{[2]}$ or the largest glycan synthesized to date a $151-\mathrm{mer},{ }^{[3]}$ where the final deprotection yields ranged from $17-54 \%$ depending on the glycan.

Similarly, we have experienced long reaction times, poor yields, and saturation of aromatic protecting groups (undesired selectivity) to their corresponding ethers in our synthesis of glycans related to Cryptococcus neoformans glucuronoxylomannan (GXM) ${ }^{[4]}$ To overcome these issues we developed a catalyst pretuning methodology that increases catalyst selectivity towards hydrogenolysis (rather than hydrogenation) and inhibits these unwanted saturation by-products. ${ }^{[4]}$ This methodology successfully tackled a major issue we faced (catalyst selectivity), however, it was still unclear to us - how and why — different palladium on carbon $(\mathrm{Pd} / \mathrm{C})$ catalysts lead to such variable results. This lack of understanding gives $\mathrm{Pd} / \mathrm{C}$ catalysts a 'black box' quality and forced extensive tedious testing with complex material, in order to identify an efficient catalyst, defined under the parameters of short reaction times, high isolated yields, and its selectivity towards hydrogenolysis over hydrogenation.

Desiring to avoid such extensive testing requirements again, we sought to advance our understanding of the key differences between commercial sources of $\mathrm{Pd} / \mathrm{C}$ in order to allow us to identify high-quality catalysts rapidly in the future. Ultimately, this could allow for prediction of a palladium on carbons quality - prior to use of valuable time and synthetic material. Furthering our understanding or what makes a $\mathrm{Pd} / \mathrm{C}$ catalyst optimal will also allow the design of more attractive heterogenous catalysts.

Here, we demonstrate clues to a palladium catalysts efficiency can be found by studying the surface chemistry of the catalysts using a combination of high-resolution TEM (HRTEM), X-ray photoelectron spectroscopy (XPS), Brunauer-Emmett-Teller (BET), and X-ray diffractometer (XRD) analysis. This work can serve as a template for others to quickly assess the quality of a catalyst at hand and circumvent the need for extensive optimization experiments with valuable materials from total synthesis. Finally, we demonstrate the analysis of the palladium catalysts completed mirrors 'the predicted activity' by comparing the catalyst performance of these samples in the deprotection of a serotype A decasaccharide. This study will inform the design of high-quality $\mathrm{Pd} / \mathrm{C}$ catalysts.

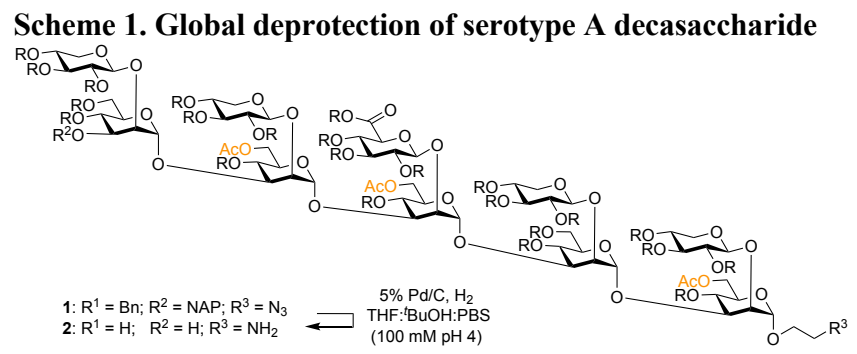


Table 1. Global Deprotection of GXM Glycan

\begin{tabular}{|c|c|c|c|c|c|c|c|}
\hline Entry & Substrate & Catalyst & Supplier & Pre-treatment & $\begin{array}{l}\text { Level of saturation } \\
\text { side-products [\%] }\end{array}$ & Time [days] & Yield [\%] \\
\hline 1 & 1 & $20 \% \mathrm{Pd}[\mathrm{OH}]_{2} / \mathrm{C}$ & Sigma-Aldrich & $\mathrm{No}^{[a]}$ & 39 & 5.5 & $66^{[\mathrm{b}]}$ \\
\hline 2 & 1 & $10 \% \mathrm{Pd} / \mathrm{C}$ & Sigma-Aldrich & $\mathrm{No}^{[a]}$ & 53 & 4 & $57^{[\mathrm{b}]}$ \\
\hline 3 & 1 & $5 \% \mathrm{Pd} / \mathrm{C}$ & Strem Chemicals & $\mathrm{No}^{[\mathrm{a}]}$ & 10 & 1.5 & $84^{[b]}$ \\
\hline 4 & 1 & $20 \% \mathrm{Pd}[\mathrm{OH}]_{2} / \mathrm{C}$ & Sigma-Aldrich & $\mathrm{Yes}^{[\mathrm{c}]}$ & 0 & 6 & 66 \\
\hline 5 & 1 & $10 \% \mathrm{Pd} / \mathrm{C}$ & Sigma-Aldrich & Yes $^{[\mathrm{c}]}$ & 0 & 5 & 58 \\
\hline 6 & 1 & $5 \% \mathrm{Pd} / \mathrm{C}$ & Strem Chemicals & $\mathrm{Yes}^{[\mathrm{c}]}$ & 0 & 2 & 88 \\
\hline
\end{tabular}

[a] untreated catalyst, EtOAc:MeOH:AcOH (4:1:1:1 v/v/v), 10 bar. [b] Combined yield of desired decasaccharide and saturated side-products. [c] Preconditioned catalyst (see protocol), THF: ${ }^{\mathrm{BuOH}}$ :PBS $(100 \mathrm{mM} \mathrm{pH} 4)(60: 10: 30 \mathrm{v} / \mathrm{v} / \mathrm{v}), 10 \mathrm{bar}$.

\section{Results and Discussion}

A key focus in our laboratory is directed towards the synthesis of glycans from C. neoformans, ${ }^{[5,6]}$ in particular the major component of its capsular polysaccharide, GXM. From native sources the GXM is highly heterogenous and to date large components of the biosynthesis are unknown - meaning synthetic glycans are currently the only viable means to access structures in any meaningful purity. ${ }^{[7]}$ With the aim of developing vaccine candidates based off these structures - we based our optimisation study using a synthetic decasaccharide 2 (Scheme 1), a current vaccine candidate in our lab. ${ }^{[8]}$

\section{Catalytic Performance of Commercial Catalysts}

Using either a Pearman's catalyst $\left(20 \% \mathrm{Pd}[\mathrm{OH}]_{2} / \mathrm{C}\right.$, SigmaAldrich) or a $10 \% \mathrm{Pd} / \mathrm{C}$ (Sigma-Aldrich) both led to exceedingly long reaction times (5-6 days) (Table 1 Entry 1 \& 2), intermediate yields (57-66\%), and high levels of saturation of aromatic protecting groups $(39-53 \%)$. While using the Evonik Noblyst ${ }^{\circledR}$ from Strem Chemicals (Table 1 Entry 3 ) we found reaction times were shortest (1.5 days), yields were highest (84\%) and low levels of aromatic saturation protecting groups $(10 \%)$.

Next we performed the hydrogenolysis reaction using our recently disclosed catalysts pre-tuning strategy. ${ }^{[4]}$ This protocol is useful as it inhibits saturation of aromatic protecting groups (e.g. benzyl and naphthylmethyl ethers). As expected, when using our protocol, no saturation of aromatic protecting groups occurred and our desired 6-O-acetylation pattern stayed intact (Table 1 Entry 36). However, the pre-tuning strategy does not shorten reaction times or impact the isolated yields of $\mathbf{2}$, which closely mirrored the nonpre-treated catalysts (Table 1). Overall, the 5\% Pd/C Evonik Noblyst $^{\mathbb{R}}$ from Strem Chemicals allowed access to the desired decasaccharide 2 in the shortest reaction times (2 days), highest yields (88\%), and no aromatic protecting group related saturation when using our pre-tuning methodology (Figure 2, Table 1 Entry 3). ${ }^{[4]}$

While pleased with our findings, we sought to further push our understanding in relation to the wide variability experienced when using different palladium on carbon catalysts. To achieve this, we sought to characterize the catalysts with a range of spectroscopic and imaging techniques. Envisaging that in the future we could predict a catalysts activity through analysis without the need for extensive optimisation reactions using precious synthetic material.

\section{Characterisation of Palladium on carbon catalysts}

\section{X-ray diffraction (XRD)}

$\mathrm{XRD}$ analysis of the $5 \% \mathrm{Pd} / \mathrm{C}$ from Strem Chemicals (Figure 1A) obtained diffraction peaks of at $2 \theta$ of $33.3^{\circ}, 34.4^{\circ}, 42.9$ and $55.3^{\circ}$ are assigned to the (002), (101), (110) and (112) facets of tetragonal PdO (powder diffraction file, PDF No. 88-2434), ${ }^{[9,10]}$ the existence of palladium was confirmed by the peaks at $2 \theta$ of $40.1^{\circ}, 46.7^{\circ}$ and $68.1^{\circ}$, which are correspondent to the (111), (200) and (220) planes of cubic Pd (PDF No. 05-0681) respectively. ${ }^{[11-13]}$ The broad and low intensities of the diffraction peaks are in the $5 \% \mathrm{Pd} / \mathrm{C}$ (Strem Chemicals) indicate that the particles are small. While the XRD pattern for the two Sigma-Aldrich catalysts shows clear peaks at $33.3^{\circ}, 34.4^{\circ}, 42.9^{\circ}$ and $55.3^{\circ}$ for crystalline tetragonal $\mathrm{PdO}$, matching well with PDF No. 88-2434. The corresponding XRD peaks intensity from the Sigma-Aldrich catalysts showed a significant increase, compared to those of the $5 \% \mathrm{Pd} / \mathrm{C}$ (Strem Chemicals), confirming the size of particles of $\mathrm{PdO}$ are larger in the Sigma-Aldrich catalysts. All of the samples showed a very broad peak located at $2 \theta$ of $\sim 25^{\circ}$, which assigns to the (002) diffraction planes of graphite microcrystals in the disordered carbon. ${ }^{[13,14]}$

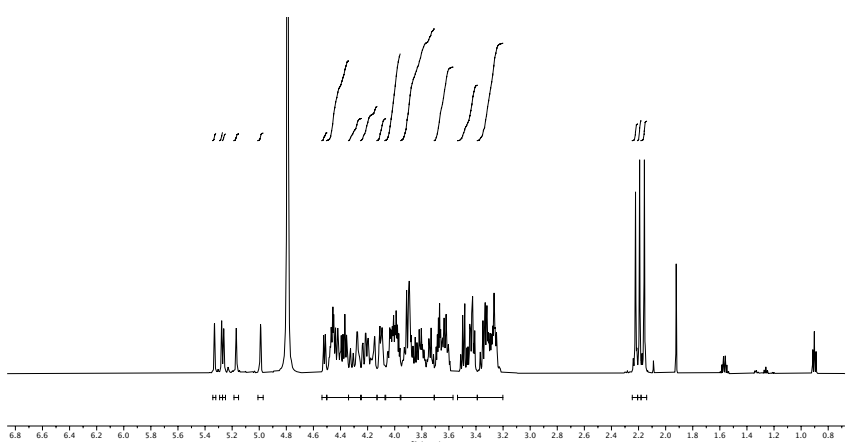

Figure 2. ${ }^{1} \mathrm{H}$ NMR Spectrum of Serotype A Decasaccharide.

The presence of large quantities of $\mathrm{PdO}\left(\mathrm{Pd}^{2+}\right)$ in the twolower quality $\mathrm{Pd} / \mathrm{C}$ catalysts (Sigma-Aldrich catalysts, Table 1 Entry 1-2 and 4-5) in combination with the larger particle sizes likely contributes to the longer reaction times required when using these catalysts. As when the $\mathrm{Pd} / \mathrm{C}$ catalyst is first exposed to the hydrogen atmosphere, it must first reduce the $\mathrm{Pd}^{2+}$ to $\mathrm{Pd}^{0}$. Meaning the oxidative addition step in the catalytic cycle cannot initially occur (meaning lower quantities of active Pd to complete hydrogenolysis). Further larger particles are well understood to affect rates of reaction and therefore also helps to explain the lower efficiency of the SigmaAldrich catalysts.

Transmission electron microscopy (TEM) 
TEM images were taken of each catalyst and enabled visualisation of the morphology and size distribution of the catalysts (Figure 1C). The $5 \% \mathrm{Pd} / \mathrm{C}$ (Strem Chemicals) indicated that Pd and $\mathrm{PdO}$ nanoparticles are uniformly dispersed on the carbon with the

A

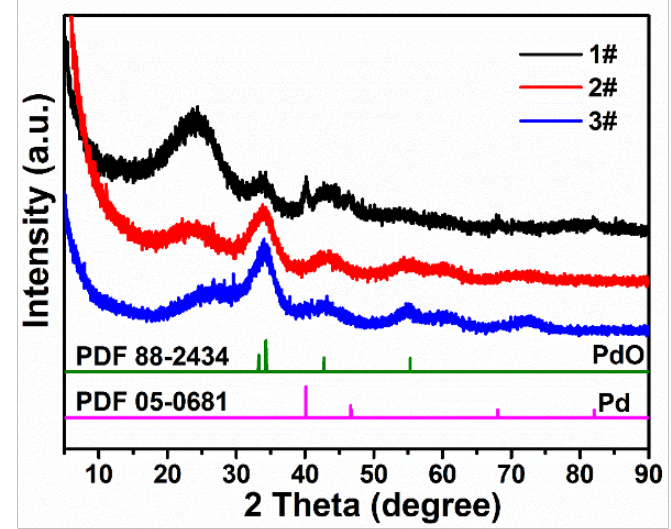

B

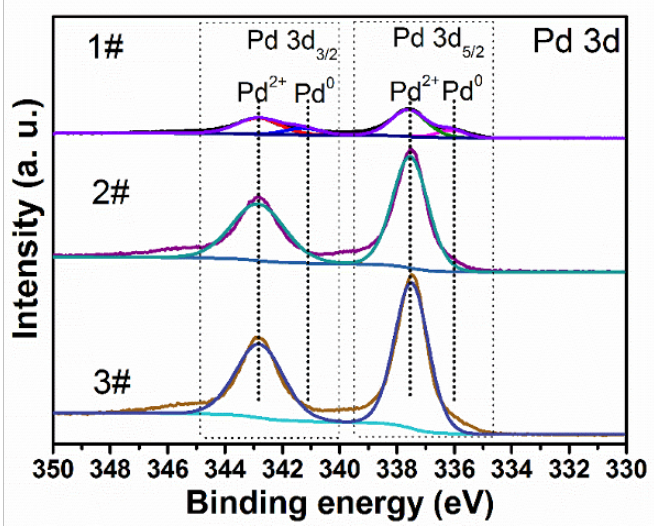

C mean size of $\sim 4 \mathrm{~nm}$. The existence of large numbers of active sites in the corners and edges of small-sized nanoparticles, is consistent with the observation of a more favourable catalytic performance during the hydrogenolysis reactions (Table 1 Entry $3 \& 6$ ).
TEM
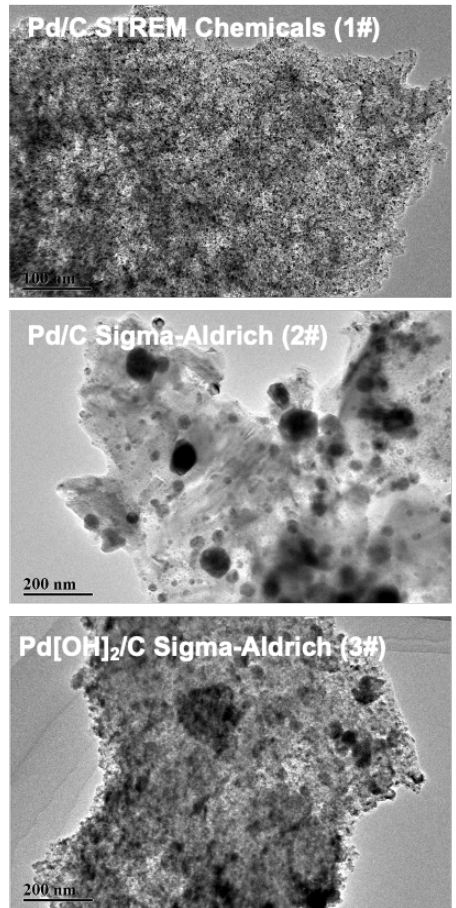

HRTEM
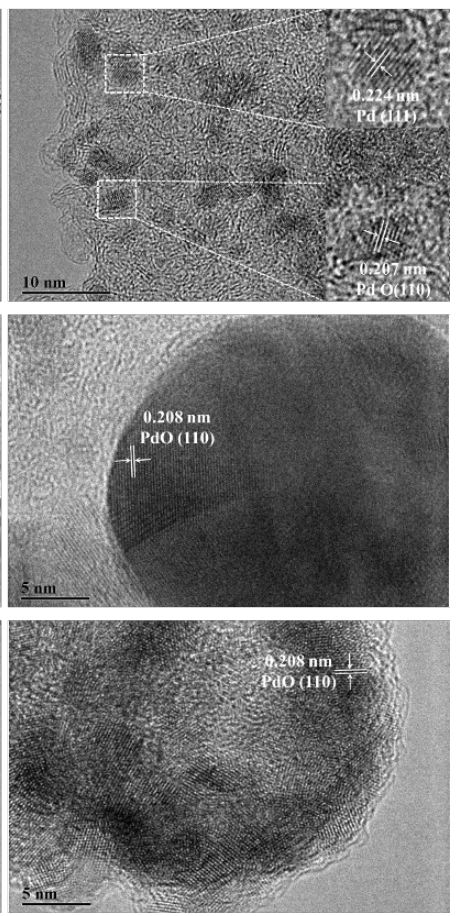

Figure 1. Characterization of Palladium on Carbon Catalysts. Pd/C STREM Chemicals (1\#), Pd/C Sigma-Aldrich (2\#), and Pd[OH] $2 / C$ Sigma-Aldrich (3\#). A. XRD patterns of the Pd/C. B. XPS for Pd 3d electrons. C. TEM and HRTEM of catalyst. Scale inset.

The high-resolution TEM (HRTEM) image of the $5 \% \mathrm{Pd} / \mathrm{C}$ (Strem Chemicals) (Figure 1C) reveals two lattice fringes with the space of $0.224 \mathrm{~nm}$ and $0.207 \mathrm{~nm}$, which correspond to the (111) crystalline plane of $\mathrm{Pd}$ and (110) crystalline plane of $\mathrm{PdO}$, respectively. This indicates the coexistence of $\mathrm{Pd}$, and $\mathrm{PdO}$ in the $5 \% \mathrm{Pd} / \mathrm{C}$ (Strem Chemicals). Conversely, the catalysts from SigmaAldrich exhibit large particle size and poor size distribution, which is not favourable for catalytic process and corresponds to the lower isolated yields and longer reaction times experienced when using these catalysts (Table 1 Entry $1 \& 2$ ). The HRTEM image of the catalysts from Sigma-Aldrich indicate that the lattice spacing of $0.208 \mathrm{~nm}$ correspond to the (110) crystal plane of PdO.

\section{X-ray photoelectron spectroscopy (XPS) analysis}

The elemental constituents and states of the catalysts were analysed using high-resolution XPS analysis (Figure 1B). Binding energies of 337.5 and $342.8 \mathrm{eV}$ were observed in all catalysts and were ascribed to $\mathrm{Pd}^{2+} 3 \mathrm{~d}_{5 / 2}$ and $3 \mathrm{~d}_{3 / 2}$ split orbitals of $\mathrm{PdO}$, respectively. Additionally, in the $5 \% \mathrm{Pd} / \mathrm{C}$ (Strem Chemicals) another two lower binding energies of 336.0 and $341.1 \mathrm{eV}$ were assigned to $3 \mathrm{~d}_{5 / 2}$ and $3 \mathrm{~d}_{3 / 2}$ levels of metallic $\operatorname{Pd}\left(\operatorname{Pd}^{0}\right)$, respectively. ${ }^{[15-17]}$ This again confirmed that both $\mathrm{PdO}$ and Pd exist in the $5 \% \mathrm{Pd} / \mathrm{C}$ (Strem Chemicals) but not in the other two catalysts.

\section{Brunauer-Emmett-Teller (BET) analysis}

In order to further verify the effect of catalyst microstructure on the catalytic performance, the specific surface area and microstructure of the catalysts were investigated by $\mathrm{N}_{2}$ adsorption/desorption experiments. Figure 3 shows the $\mathrm{N}_{2}$ adsorption/desorption isotherm linear plots for the acquired catalysts. The isotherms display a typical type IV behaviour, with a sharp uptake at low relative pressure, which is distinctive for mesoporous materials, suggesting that plenty of mesopores exist in these catalysts, this conclusion is also supported by the TEM characterization. The Brunauer-Emmett-Teller (BET) specific surface area of the sample $5 \% \mathrm{Pd} / \mathrm{C}$ Strem Chemicals (1\#), $10 \%$ $\mathrm{Pd} / \mathrm{C}$ Sigma-Aldrich (2\#), and $20 \% \mathrm{Pd}[\mathrm{OH}]_{2} / \mathrm{C} \mathrm{(3 \# )} \mathrm{is} \mathrm{about} 897.3$ $\mathrm{m}^{2} / \mathrm{g}, 898.2 \mathrm{~m}^{2} / \mathrm{g}$ and $778.3 \mathrm{~m}^{2} / \mathrm{g}$, respectively.

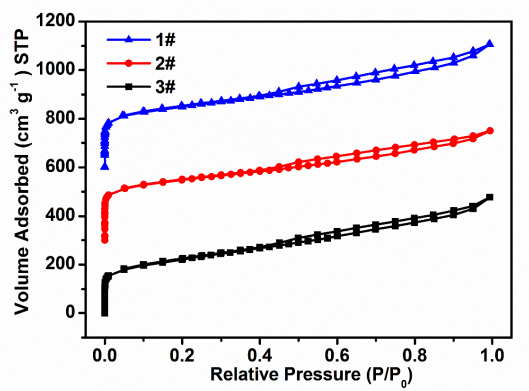

Figure 3. $\mathrm{N}_{2}$ adsorption-desorption isotherms of sample Pd/C STREM Chemicals (1\#), $\mathrm{Pd} / \mathrm{C}$ Sigma-Aldrich (2\#), and $\mathrm{Pd}[\mathrm{OH}]_{2} / \mathrm{C}$ Sigma-Aldrich (3\#). 


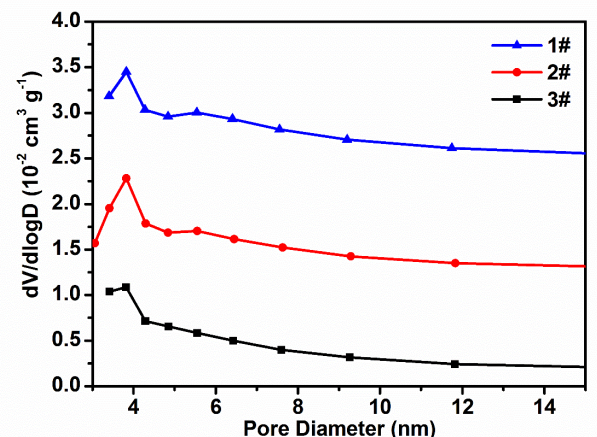

Figure 4. Pore size distribution plots of samples Pd/C STREM Chemicals (1\#), Pd/C Sigma-Aldrich (2\#), and Pd[OH $]_{2} / C$ Sigma-Aldrich (3\#).

The average pore size distribution (Figure 4) emphasized the presence of mesopores with diameters centred at about $\sim 4.0 \mathrm{~nm}$. It can be seen from the above microstructure data that the specific surface area and pore size distribution of the catalyst are not obvious different, so the significant difference of catalytic performance reflected by the catalyst mainly comes from the different composition of its active substance ( $\mathrm{Pd}$ and $\mathrm{PdO})$.

\section{Conclusions}

We completed the analysis of three sources of palladium on carbon catalysts physical and chemical properties - demonstrating the wide variability in quality from commercial suppliers. These techniques could be used to predict a catalysts efficiency prior to the use of precious synthetic material. These findings are of particular importance to chemists in both industry and academia where identifying high-quality catalysts improves efficiency in terms of both access to desired target compounds and time used in optimisation studies. This study will enable steps towards the design of higher-quality $\mathrm{Pd} / \mathrm{C}$ catalysts.

\section{Experimental Section}

\section{Synthesis of decasaccharide substrate}

To complete the total synthesis of the serotype A decasaccharide we followed a convergent building block approach, utilizing di- and tetrasaccharide thioglycoside building blocks. ${ }^{[18]}$ The synthesis of which has reported previously, ${ }^{[8]}$ the use of a convergent synthesis is attractive for several reasons; as it allows quick assembly of the target GXM glycans in minimal steps; the 6-O-acetylation along the mannose backbone is preinstalled; and glycan branching is formed at an early stage, specifically the $\beta-1,2$ xylose branches and $\beta-1,2$ glucuronic acid branches.

\section{Procedure for Catalyst Pre-treatment ${ }^{[4]}$}

$500 \mathrm{mg} \mathrm{Pd} / \mathrm{C}$ (any commercial catalyst), was suspended in $1 \mathrm{~mL}$ DMF: $\mathrm{H}_{2} \mathrm{O}$ mixture $(80: 20 \mathrm{v} / \mathrm{v})$, and the solution was made acidic by the addition of $200 \mu \mathrm{L} \mathrm{HCl}$ (ACS Reagent, 37\%, pH 2-3), with or without an atmosphere of hydrogen gas for about 20 minutes. The presence of dimethylamine was confirmed via ninhydrin staining. The treated $\mathrm{Pd} / \mathrm{C}$ catalysts was re-isolated though filtration. The moistened catalyst was then be used directly in the hydrogenolysis reaction.

\section{Optimised Procedure for hydrogenolysis reaction ${ }^{[4]}$}

The treated catalyst (0.2-0.5 molar eq. of palladium per benzyl group) was added to a solution of oligosaccharide (1 eq.) dissolved in THF:tert-butyl alcohol:PBS (100 mM, pH 4) (60:10:30, v/v/v). The reaction was placed in a high pressure reactor at $10 \mathrm{bar}$ and was monitored via normal phase TLC (MeCN:H2O mixtures) and MALDI-TOF mass spectrometry Once complete the reaction mixture was filtered through a plug of Ceilte ${ }^{\circledR}$ and then concentrated in vacuo. The residue was then re-dissolved in a minimal amount of sterile water and purified with a Bio-gel P2 Column, after lyophilization to yield the desired product.

\section{Palladium on Carbon Characterization}

A transmission electron microscope (JEOL JEM-2100F) was used to obtain transmission electron microscopy (TEM) images, highresolution TEM (HRTEM) images at an acceleration voltage of 200 $\mathrm{kV}$. An X-ray diffractometer (XRD, Bruker D8 Advance) with Cu$\mathrm{K} \alpha$ radiation was used for analysing the crystallographic structure of the as-prepared samples from $5^{\circ} \sim 90^{\circ}$ with a scanning step of $0.02^{\circ}$. The surface elemental composition and chemical state of the asprepared samples were collected from an X-ray photoelectron spectra spectrometer (XPS, Kratos AXIS ULTRA DLD), in which a monochromatic $\mathrm{Al} \mathrm{K} \alpha$ source $(\mathrm{h} v=1486.6 \mathrm{eV})$ was applied. All binding energies were calibrated using the $\mathrm{C} 1 \mathrm{~s}$ hydrocarbon peak at $284.60 \mathrm{eV}$.

\section{General Notes}

Silica gel flash chromatography was carried out using automated flash chromatography systems, Buchi Reveleris ${ }^{\circledR}$ X2 (UV 200-500 $\mathrm{nm}$ and ELSD detection, Reveleris ${ }^{\circledR}$ silica cartiges $40 \mu \mathrm{m}$, BÜCHI Labortechnik AG). Size-exclusion chromatography was performed on Bio-Gel ${ }^{\circledR}$ P-2 (Bio-Rad Laboratories Inc.) using isocratic elution $\left(\mathrm{H}_{2} \mathrm{O}: \mathrm{tBuOH}, 99: 1, \mathrm{v} / \mathrm{v}\right)$. Instrumentation: peristaltic pump P-3 (Pharmacia Fine Chemicals), refractive index detector Iota 2 (Precision Instruments), PrepFC fraction collector (Gilson Inc.). Software: Trilution ${ }^{\circledR}$ LC (version 1.4, Gilson Inc.). All chemicals for the synthesis were purchased from commercial suppliers (Acros, Carbosynth Ltd, Fisher Scientific Ltd, A/S, Merck, Sigma-Aldrich, VWR, Strem Chemicals and AlfaAesar) and used without purification. Dry Solvents were obtained from a PureSolv-ENTM solvent purification system (Innovative Technology Inc.). All other anhydrous solvents were used as purchased from Sigma-Aldrich in AcroSeal ${ }^{\circledR}$ bottles.

\section{Acknowledgements}

We thank Dr Yannick Ortin and Dr Jimmy Muldoon for NMR and MS support. C.J.C. was funded by Irish Research Council postgraduate award (GOIPG/2016/998). S.O was supported by Science Foundation Ireland Award 13/IA/1959.

Keywords: heterogenous catalysis $\bullet$ hydrogenolysis $\bullet$ palladium • global deprotections $\bullet$ glycans $\bullet$

[1] Q. Zhu, Z. Shen, F. Chiodo, S. Nicolardi, A. Molinaro, A. Silipo, B. Yu, Nat. Commun. 2020, 11, 1-7.

[2] M. Guberman, M. Bräutigam, P. H. Seeberger, Chem. Sci. 2019, 10, 5634-5640. 
[3] A. A. Joseph, A. Pardo-Vargas, P. H. Seeberger, J. Am. Chem. Soc. 2020, 142, 8561-8564.

[4] C. Crawford, S. Oscarson, European J. Org. Chem. 2020, 3332-3337.

[5] C. Crawford, M. P. Wear, D. F. Q. Smith, C. D’Errico, A. Casadevall, S. Oscarson, ChemRxiv 2020, DOI https://doi.org/10.26434/chemrxiv.12144699.v1.

[6] C. J. Crawford, S. Oscarson, Carbohydr. Res. 2020, 108150.

[7] C. J. Crawford, R. J. B. Cordero, L. Guazzelli, M. P. Wear, A. Bowen, S. Oscarson, A. Casadevall, J. Biol. Chem. 2020, 295, 4327-4340.

[8] L. Guazzelli, C. J. Crawford, R. Ulc, A. Bowen, O. McCabe, A. J. Jedlicka, M. P. Wear, A. Casadevall, S. Oscarson, Chem. Sci. 2020, 11, 9209-9217.

[9] W. Hu, G. X. Li, J. J. Chen, F. J. Huang, Y. Wu, S. D. Yuan, L. Zhong, Y. Q. Chen, Chem. Commun. 2017, 53, 6160-6163.

[10] H. J. Cho, V. T. Chen, S. Qiao, W. T. Koo, R. M. Penner, I. D. Kim, ACS Sensors 2018, 3, 2152-2158.
[11] J. Zhao, W. Jing, T. Tan, X. Liu, Y. Kang, W. Wang, New J. Chem. 2020, 44, 4604-4612.

[12] P. Xi, F. Chen, G. Xie, C. Ma, H. Liu, C. Shao, J. Wang, Z. Xu, X. Xu, Z. Zeng, Nanoscale 2012, 4, 5597-5601.

[13] L. Lan, F. Du, C. Xia, RSC Adv. 2016, 6, 109023-109029.

[14] W. Xu, C. Lv, Y. Zou, J. Ren, X. She, Y. K. Zhu, Y. Zhang, S. Chen, X. Yang, T. Zhan, et al., J. Power Sources 2019, 442, 227184.

[15] S. Meher, R. K. Rana, Green Chem. 2019, 21, 2494-2503.

[16] J. Lv, S. Wu, Z. Tian, Y. Ye, J. Liu, C. Liang, J. Mater. Chem. A 2019, 7, 12627-12634.

[17] L. Han, Q. Li, S. Chen, W. Xie, W. Bao, L. Chang, J. Wang, Sci. Rep. 2017, 7, DOI 10.1038/s41598-01707802-8.

[18] L. Guazzelli, R. Ulc, L. Rydner, S. Oscarson, Org. Biomol. Chem. 2015, 13, 6598-6610. 